\title{
An LMI-Based Controller for the Glucose-Insulin System
}

\author{
P. Latafat* \\ P. Palumbo* \\ P. Pepe* \\ L. Kovács \\ S. Panunzi \\ A. De Gaetano
}

\begin{abstract}
Exogenous insulin administration is the standard way to regulate hyperglycemia in diabetic patients and, in the recent decades, the challenging task to design an artificial pancreas has been addressed with the aim to synthesize a closedloop control law by means of sampled glucose measurements. Model-based control law allow to explicitly exploit the glucoseinsulin mathematical model, but need to cope with different sources of uncertainties and disturbances affecting the system. The present note investigates the framework of the $H_{\infty}$ control as a tool to attenuate the effect of a meal, modeled as an unknown disturbance. To this end an LMI-based feedback control law is synthesized, by properly exploiting a Delay Differential Equation model of the glucose-insulin system, that makes use of only glucose measurements, to avoid the use of insulin measurements, known to be slower and more cumbersome to obtain, more expensive and also less accurate than glucose measurements. It is shown by simulations that, besides to regulate plasma glycemia onto a desired level starting from a hyperglycemic state, the control law efficiently constrains the post-prandial increase of glycemia on a very tight control, preventing dangerous oscillations.
\end{abstract}

Index Terms - Linear Matrix Inequalities, Retarded Systems, Glucose-Insulin System.

\section{INTRODUCTION}

Diabetes Mellitus is a very common disease characterized by hyperglycemia resulting from defects in insulin secretion, insulin action, or both. The standard fashion to cope with any malfunction of the endogenous insulin feedback action is via exogenous insulin administration, usually by means of subcutaneous or intravenous insulin injections. Type 1 diabetic patients require insulin to survive, since their pancreas does not produce insulin any more; on the other hand, exogenous insulin therapies for type 2 diabetes aim to complement the endogenous, though insufficient, insulin delivery.

The present work proposes a model-based, feedback control law for an artificial pancreas, with exogenous insulin delivered intravenously. Even if directly applicable, so far, only to problems of glycemia stabilization in critically ill subjects, such as in surgical Intensive Care Units after major procedures, the use of intravenous insulin administration

* The first three authors equally contributed to the manuscript. P. Palumbo, S. Panunzi and A. De Gaetano are with the Istituto di Analisi dei Sistemi e Informatica, Italian National Research Research Council, Via dei Taurini 19, Roma, Italy. P. Latafat and P. Pepe are with the Department of Information Engineering, Computer Science, and Mathematics, University of L'Aquila, Via Gronchi 18, 67100 L'Aquila, Italy. L. Kovács is with John von Neumann Faculty of Informatics, Óbuda University, Budapest, Hungary. E-Mail Addresses: puya.latafat@mathmods.eu, pasquale.palumbo@iasi.cnr.it, pierdomenico.pepe@univaq.it,_kovacs.levente@nik.uni-obuda.hu, simona.panunzi@iasi.cnr.it, andrea.degaetano@iasi.cnr.it provides a wider range of possible strategies with respect to the subcutaneous route, and ensures a rapid delivery with negligible delays.

The glucose-insulin model chosen to synthesize the closed-loop control is a Delay Differential Equation (DDE) nonlinear system, [19], [24]. Differently from other modelbased approaches, which make use of Ordinary Differential Equation (ODE) models of the glucose-insulin system to design the feedback control law according to different control strategies (e.g. Model Predictive Control in [8], [16], Parametric Programming in [5], $H_{\infty}$ control in [25], [13], [14], [15], non-standard $H_{\infty}$ control in [3], [29]), a DDE model based control law is able to take into account irregularly varying pancreatic Insulin Delivery Rate (IDR) ([17] and references therein), thus allowing the construction of a control scheme applicable also to Type 2 diabetes patients. Despite the attention given to DDE models of the glucoseinsulin system in the last decade, their use as components of the artificial pancreas has only recently sparked interest, and has been mainly limited to open-loop approaches (see [9] and references therein). Indeed, attempts to design closedloop ODE model-based glucose controls have been limited so far to Type 1 diabetic patients (who have essentially no endogenous insulin production), circumventing in this way the need to model pancreatic IDR. The DDE model considered here can realistically account for endogenous IDR, thereby modeling in a unified fashion healthy subjects, insulin resistant and insulin-deficient diabetic patients. Such a model has already been exploited in [20], [21], [23] to track a desired glucose profile according to a control strategy based on feedback linearization with delay cancelation, with the control law synthesized for a patient at rest. In [22] it has been shown that the resulting closed-loop system satisfies the local Input-to-State Stability (ISS) property with respect to an unknown disturbance in the glucose dynamics, like a meal.

The present note investigates the synthesis of a robust glucose controller according to the $H_{\infty}$ approach, with the goal of minimizing the effect of an unknown disturbance (the meal) on the output of the system (plasma glycemia). Since a standard $H_{\infty}$ approach is developed for linear systems, we linearize the DDE nonlinear model at different levels of glycemia and insulinemia, and provide a stabilizer for each linear model by involving LMIs (see, for instance, [1], [7], [18]). Also, for each linear model we provide an observer, in order to compensate for missing insulin measurements, known to be slower and more cumbersome to obtain, more expensive and less accurate than glucose measurements.

The simulations show a very good performance of the proposed controller (i.e, the glycemia is driven to the desired 
level in good timing and satisfactory shape, avoiding dangerous oscillations). Moreover, when an unknown disturbance appears (i.e., a meal), the performance of the controller is still satisfactory, as shown by comprehensive simulations.

The paper is organized as follows. Soon after a short set of notations, some basic results on LMI-based control are briefly reported in Section II for linear time-delay systems. Section III reports the DDE model of the glucose-insulin system under investigation and Section IV details the proposed LMI-based control algorithm. Numerical simulations are reported in Section $\mathrm{V}$, followed by concluding remarks.

\section{Notations}

For a positive integer $n$, for a positive real $\Delta$ (maximum involved time-delay), $\mathcal{C}$ denotes the space of the continuous functions mapping $[-\Delta, 0]$ into $\mathbb{R}^{n}$. For a function $x$ : $[-\Delta, c) \rightarrow \mathbb{R}^{n}$, with $0<c \leq+\infty$, for any real $t \in[0, c): x_{t}$ is the function in $\mathcal{C}$ defined as $x_{t}(\tau)=x(t+\tau), \tau \in[-\Delta, 0]$. For a symmetric matrix $P \in \mathbb{R}^{n \times n}$, we say $P>0$ if $P$ is positive definite, $P<0$ if it is negative definite.

RFDE stands for retarded functional differential equation, GAS stands for uniform global asymptotic stability or uniformly, globally asymptotically stable, LMI stands for Linear Matrix Inequality. We recall here that a system is said to be 0 -GAS if the origin is GAS.

\section{PREliminaries: LMI-BASED CONTROL}

Let us consider a single-input, single-output system described by the following linear RFDE

$$
\begin{aligned}
& \dot{x}(t)=A_{0} x(t)+A_{1} x(t-\Delta)+B u(t)+B_{1} d(t), \\
& \begin{array}{lc}
x(\tau)=x_{0}(\tau), & t \geq 0, \\
y(t)=C x(t), & t \geq 0,0],
\end{array}
\end{aligned}
$$

where $x_{t} \in \mathcal{C}, t \geq 0$, is the state of the system, $u(t) \in \mathbb{R}$ is the control input, $d(t) \in \mathbb{R}$ is the unknown disturbance (Lebesgue square integrable in $\mathbb{R}^{+}$), $y(t) \in \mathbb{R}$ is the measured output, $\Delta$ is a positive real, and matrices $A_{0}, A_{1} \in$ $\mathbb{R}^{n \times n}, B, B_{1} \in \mathbb{R}^{n \times 1}, C \in \mathbb{R}^{1 \times n}$.

The proposed control law makes use of the following standard observer-based controller for the retarded linear system described by (1):

$$
\begin{aligned}
& \dot{\xi}(t)=A_{0} \xi(t)+A_{1} \xi(t-\Delta)+L(y(t)-C \xi(t))+B u(t), \quad t \geq 0, \\
& \xi(\tau)=\xi_{0}(\tau), \quad \tau \in[-\Delta, 0], \quad \xi_{0} \in \mathcal{C}, \\
& u(t)=K \xi(t), \quad t \geq 0,
\end{aligned}
$$

where $\xi(t) \in \mathbb{R}^{n}, t \geq-\Delta$, is the observed state, and $K \in \mathbb{R}^{1 \times n}, L \in \mathbb{R}^{n \times 1}$ the control and observer gains, respectively, whose design is based on the following result (see, e.g., [28], [1]; the formalism is taken from Theorem 5.6 of [1] for the design of the control gain $K$ within the state feedback $H_{\infty}$ formulation, and from Theorem 3.1 of [1] for the design of the observer gain $L$ ).
Theorem 1: Consider system (1). Let the following hypotheses hold true:

- there exist symmetric, positive definite matrices $P>0$, $Q>0$, in $\mathbb{R}^{n \times n}$, and a vector $Z \in \mathbb{R}^{n}$, such that the following LMI holds satisfied

$$
\left[\begin{array}{cc}
A_{0}^{T} P+P A_{0}-C^{T} Z^{T}-Z C+Q & P A_{1} \\
A_{1}^{T} P & -Q
\end{array}\right]<0
$$

- Let $\gamma>0$ be a given scalar. Let the symmetric, positivedefinite matrices $X, Q_{i} \in \mathbb{R}^{n \times n}, i=1,2,3$, and a vector $Y \in \mathbb{R}^{1 \times n}$ such that the following LMI holds satisfied

$$
\left[\begin{array}{cccc}
H_{1,1} & B_{1} & H_{1,3} & H_{1,4} \\
B_{1}^{T} & -\gamma^{2} I_{n}+\Delta B_{1}^{T} Q_{3} B_{1} & 0 & 0 \\
H_{1,3}^{T} & 0 & -I_{n} & 0 \\
H_{1,4}^{T} & 0 & 0 & -Q
\end{array}\right]<0
$$

where $I_{n}$ is the identity matrix in $\mathbb{R}^{n \times n}$, and

$$
\begin{aligned}
& H_{1,1}=\left(A_{0}+A_{1}\right) X+X\left(A_{0}+A_{1}\right)^{T} \\
& +B Y+Y^{T} B^{T}+\Delta A_{1} Q_{1} A_{1}^{T}+\Delta A_{1} Q_{2} A_{1}^{T}, \\
& H_{1,3}=X C^{T} \text {, } \\
& H_{1,4}=\left[\begin{array}{lll}
X A_{0}^{T}+Y B^{T} & X A_{1}^{T} & A_{1}
\end{array}\right], \\
& Q=\frac{1}{\Delta}\left[\begin{array}{ccc}
Q_{1} & 0 & 0 \\
0 & Q_{2} & 0 \\
0 & 0 & Q_{3}
\end{array}\right] \text {. }
\end{aligned}
$$

Then, the observer-based control law (2), with $L=P^{-1} Z$, $K=Y X^{-1}$, is such that the closed-loop system (1), (2) is, in the disturbance-free case, 0-GAS. Moreover, for the system (1) in closed-loop with the control law $u(t)=K x(t)$, the $H_{\infty}$ inequality holds (when the initial state $x_{0}$ is assumed equal to zero):

$$
\left\|y_{[0, \infty)}\right\|_{2} \leq \gamma\left\|d_{[0, \infty)}\right\|_{2}
$$

Remark 2: In the disturbance free case (i.e., $d(t) \equiv 0$ ), when the observer-based control law (2) is applied to the system described by (1), we obtain, for the estimation error $e(t)=x(t)-\xi(t)$, the equation

$$
\dot{e}(t)=\left(A_{0}-L C\right) e(t)+A_{1} e(t-\Delta),
$$

and for the variable $x(t)$ the equation

$$
\dot{x}(t)=\left(A_{0}+B K\right) x(t)+A_{1} x(t-\Delta)-B K e(t) .
$$

If the LMI (3) is feasible and $L$ is chosen as $L=P^{-1} Z$, then the system described by (7) is 0-GAS. If the LMI (4) is feasible, and $K$ is chosen as $K=Y X^{-1}$, then the system described by (8) is 0-GAS (i.e., when $e(t) \equiv 0$ ). The system described by (7), (8) is in cascade form. From linearity and the 0-GAS property, it follows that the system described by (8) is input-to-state stable (state $x$, input $e$, see [26], Proposition 2.5). Thus the cascade (7), (8) is 0GAS (see [10], Remark 13, and take into account of converse Lyapunov-Krasovskii theorems in [11], [12]). It follows that the closed-loop system (1), (2) (which can be rewritten in the 
cascade form (7), (8)) is, in the disturbance free-case, 0-GAS. The same conclusion can be easily achieved by rewriting the RFDEs (7), (8) on a suitable Hilbert space (see [6] and references therein), and exploiting the exponential stability of involved semigroups as well as equivalence of the 0-GAS property with respect to continuous or absolutely continuous initial conditions (see [27]).

Remark 3: In the disturbance free-case, if one sets $B_{1}=0$ in the LMI (4), the positive real $\gamma$ plays no role. That is, the LMI (4) with $B_{1}=0$ is feasible if and only if the following LMI (with the same entries as in (5)) is feasible

$$
\left[\begin{array}{ccc}
H_{1,1} & H_{1,3} & H_{1,4} \\
H_{1,3}^{T} & -I_{n} & 0 \\
H_{1,4}^{T} & 0 & -Q
\end{array}\right]<0
$$

In this paper we will find the controller by always using the LMI (4) with $B_{1} \neq 0$, since the 0-GAS property is guaranteed by the LMI (4), when feasible with $B_{1} \neq 0$. Moreover, we will make use of the observer-based control law (2), for which only the 0-GAS property of the closedloop disturbance free system is guaranteed, also in the case the disturbance is not zero, and we will show excellent performances with the practical case study dealt within this paper.

\section{The Glucose-Insulin System}

Denote $G(t),[\mathrm{mM}], I(t),[\mathrm{pM}]$, plasma glycemia and insulinemia, respectively. The glucose-insulin model considered here belongs to the family of DDE models described in [19] and consists of a single discrete-delay differential equation system:

$$
\begin{aligned}
& \frac{d G(t)}{d t}=-K_{x g i} G(t) I(t)+\frac{T_{g h}}{V_{G}}+\frac{d(t)}{V_{G}}, \quad t \geq 0, \\
& \frac{d I(t)}{d t}=-K_{x i} I(t)+\frac{T_{i G \max }}{V_{I}} f\left(G\left(t-\tau_{g}\right)\right)+\frac{u(t)}{V_{I}},
\end{aligned}
$$

with the nonlinear function $f(\cdot)$ modeling the pancreatic Insulin Delivery Rate as:

$$
f(G)=\frac{\left(\frac{G}{G^{*}}\right)^{\gamma}}{1+\left(\frac{G}{G^{*}}\right)^{\gamma}}
$$

Refer to [24] for details on the model parameters. The signal $u(t)[\mathrm{pmol} / \mathrm{kgBW} / \mathrm{min}]$ is the exogenous intra-venous insulin delivery rate, i.e. the control input; $d(t)$ [mmol $/ \mathrm{kgBW} / \mathrm{min}]$ is an unknown disturbance, which models a glucose intake, e.g. by means of a meal.

Initial conditions are $G(\tau)=G_{0}(\tau), I(\tau)=I_{0}(\tau)$, $\tau \in\left[-\tau_{g}, 0\right]$, corresponding to the plasma glucose/insulin concentrations before the control input $u(t)$ is applied and the disturbance $d(t)$ is administered. For instance, they can be assumed equal to the constant basal levels $\left(G_{b}, I_{b}\right)$.

It has to be stressed that model (10) may represent equally well healthy subjects and insulin-resistant or severely insulindeficient diabetic patients by appropriately changing the parameter values.

\section{THE LMI-BASED CONTROL ALGORITHM}

Let $G_{d e s}[\mathrm{mM}]$ be the desired level of plasma glycemia, smaller than the basal hyperglycemic level of glycemia of a diabetic patient, and define

$$
\begin{aligned}
I_{\text {des }} & =\frac{T_{g h}}{V_{G}} \frac{1}{K_{x g i} G_{\text {des }}}, \\
u_{\text {des }} & =V_{I} K_{x i} I_{\text {des }}-T_{i G \max } f\left(G_{\text {des }}\right)
\end{aligned}
$$

Then, if $G(\tau)=G_{d e s}, \tau \in\left[-\tau_{g}, 0\right], I(0)=I_{d e s}, u(t)=$ $u_{d e s}, t \geq 0, d(t) \equiv 0$, the solution of (10) is given by $G(t)=$ $G_{\text {des }}, I(t)=I_{\text {des }}, t \geq 0$. In other words, once $G_{\text {des }}$ has been chosen, we may compute $I_{d e s}$ and $u_{d e s}$ from (12) as the insulinemia and the input references which asymptotically correspond to a perfect tracking of $G_{\text {des }}$ [21].

Let $G_{\max }>G_{b}$ be a feasible maximum value for plasma glycemia (indeed, from a mathematical viewpoint such a bound exists since it has been proven that the DDE model (10) has persistent solutions, [19]) and define the following partition of the glucose interval $\left(0, G_{\max }\right]$ :

$$
W_{k}=\left(G_{k}-a_{k}, G_{k}+b_{k}\right], \quad k=0,1, \ldots, p
$$

with $G_{0}=0, G_{p}=G_{\max }, a_{0}=0, b_{p}=0$ and

$$
\begin{gathered}
0 \leq G_{k}<G_{k+1} \leq G_{\max } \quad \forall k=0,1, \ldots, p-1 \\
a_{k}=\frac{1}{2}\left(G_{k}-G_{k-1}\right), \quad k=1,2, \ldots, p \\
b_{k}=\frac{1}{2}\left(G_{k+1}-G_{k}\right), \quad k=0,1, \ldots, p-1
\end{gathered}
$$

For each $G_{k}, k=0,1, \ldots, p$, the following values can be computed for $I_{k}$ and $u_{k}$ :

$$
\begin{aligned}
I_{k} & =\frac{T_{g h}}{V_{G}} \frac{1}{K_{x g i} G_{k}}, \\
u_{k} & =V_{I} K_{x i} I_{k}-T_{i G_{\max }} f\left(G_{k}\right)
\end{aligned}
$$

The proposed control algorithm consists of a variable structure regulator with the control parameters tuned according to the linearization of the DDE nonlinear model of the glucose-insulin system around the triple $\left(G_{k}, I_{k}, u_{k}\right)$ :

$$
\dot{x}_{k}(t)=A_{0, k} x_{k}(t)+A_{1, k} x_{k}\left(t-\tau_{g}\right)+B v_{k}(t)+B_{1} d(t)
$$

where

$$
x_{k}(t)=\left[\begin{array}{c}
G(t)-G_{k} \\
I(t)-I_{k}
\end{array}\right] \quad v_{k}(t)=u(t)-u_{k}
$$

and

$$
\begin{aligned}
& A_{0, k}=\left[\begin{array}{cc}
-K_{x g i} I_{k} & -K_{x g i} G_{k} \\
0 & -K_{x i}
\end{array}\right], \quad B=\left[\begin{array}{c}
0 \\
1 / V_{I}
\end{array}\right] \\
& A_{1, k}=\left[\begin{array}{cc}
0 & 0 \\
\frac{T_{i G \max }}{V_{I}} f^{\prime}\left(G_{k}\right) & 0
\end{array}\right], \quad B_{1}=\left[\begin{array}{c}
1 / V_{G} \\
0
\end{array}\right]
\end{aligned}
$$

Without loss of generality, assume $G_{d e s}=G_{\bar{k}}<G_{b}$ for some $\bar{k} \in\{1,2, \ldots, p-1\}$. The idea of the proposed methodology is to move towards the desired level of glycemia by means of intermediate control steps, according to which, at each step the controller aims to track a temporary desired glycemia given by a suitably chosen $G_{k} \in W_{k}$ : to this 
end Theorem 1 is repeatedly applied to the DDE system linearized around the triple $\left(G_{k}, I_{k}, u_{k}\right)$. Thus, these triples constitute an a priori setting of the control algorithm. As a matter of fact, the matrices of the linearized system can be computed offline, as well as the control gains $K$ and the observer gains $L$ (as feasible LMI solutions), as a sort of control library to be properly selected during the algorithm application. To this aim, assume there exist LMI solutions to (3)-(4) for any region $W_{k}$ of the partition, and define $\left(K_{k}, L_{k}\right)$ a chosen pair of gains associated to $W_{k}$. The algorithm follows below.

Algorithm. Define matrix $C=\left[\begin{array}{ll}1 & 0\end{array}\right]$. Let $\widehat{G}(\tau)=$ $\widehat{G}_{0}(\tau), \widehat{I}(\tau)=\widehat{I}_{0}(\tau), \tau \in\left[-\tau_{g}, 0\right]$, with $\widehat{G}_{0}, \widehat{I}_{0}$ continuous functions (for instance, constant) mapping $\left[-\tau_{g}, 0\right] \mapsto \mathbb{R}$, initial estimations of the glucose and insulin variables. Let $G(0) \in W_{j_{0}}$, with $j_{0} \in\{0,1, \ldots, p\}$. Let

$$
l=\left\{\begin{array}{cc}
j_{0}+1, & j_{0}<\bar{k}, \\
j_{0}-1, & j_{0}>\bar{k}, \\
j_{0}, & j_{0}=\bar{k}
\end{array}\right.
$$

Let $j \in\{1,2, \ldots, p-1\}$ be a switching index, as specified in next points, and consider the closed-loop system given by (10) and $u(t)=v(t)+u_{j}$ as follows:

$$
\begin{aligned}
\dot{\xi}(t)= & A_{0, j} \xi(t)+A_{1, j} \xi\left(t-\tau_{g}\right) \\
& +L_{j}\left(G(t)-G_{j}-C \xi(t)\right)+B v(t), \quad t \geq 0, \\
\xi(\tau)= & {\left[\begin{array}{c}
\widehat{G}(\tau)-G_{l} \\
\widehat{I}(\tau)-I_{l}
\end{array}\right], } \\
v(t)=K_{j} \xi(t), & \tau \in\left[-\tau_{g}, 0\right],
\end{aligned}
$$

- if $G(t) \in W_{k}$, with $k>\bar{k}$, then $j=k-1,\left(K_{j}, L_{j}\right)=$ $\left(K_{k-1}, L_{k-1}\right)$ and

$$
A_{0, j}=A_{0, k-1}, \quad A_{1, j}=A_{1, k-1},
$$

with $A_{0, k-1}, A_{1, k-1}$ computed according to the linearization of the DDE system around the triple $\left(G_{k-1}, I_{k-1}, u_{k-1}\right),(20)-(21)$;

- if $G(t) \in W_{k}$, with $k<\bar{k}$, then $j=k+1,\left(K_{j}, L_{j}\right)=$ $\left(K_{k+1}, L_{k+1}\right)$ and

$$
A_{0, j}=A_{0, k+1}, \quad A_{1, j}=A_{1, k+1},
$$

with $A_{0, k+1}, A_{1, k+1}$ computed according to the linearization of the DDE system around the triple $\left(G_{k+1}, I_{k+1}, u_{k+1}\right),(20)-(21)$;

- if $G(t) \in W_{k}$, with $k=\bar{k}$, then $j=\bar{k},\left(K_{j}, L_{j}\right)=$ $\left(K_{\bar{k}}, L_{\bar{k}}\right)$ and

$$
A_{0, j}=A_{0, \bar{k}}, \quad A_{1, j}=A_{1, \bar{k}},
$$

with $A_{0, \bar{k}}, A_{1, \bar{k}}$ computed according to the linearization of the DDE system around the triple $\left(G_{d e s}, I_{d e s}, u_{d e s}\right)$, (20)-(21).

Remark 4: Notice that the control law is designed without taking into account saturation constraints that, on the contrary, are clearly present since the control law is, in fact, an insulin infusion rate and cannot become negative. To properly cope with this fact, the algorithm trivially provides a zero
TABLE I

CONTROL/OBSERVER PARAMETERS FOR PATIENT AT REST

\begin{tabular}{|c|c|c|c|}
\hline Regions & \multicolumn{2}{|c|}{$K$} & \multicolumn{2}{|c|}{$L$} \\
\hline$W_{10}$ & {$\left[\begin{array}{ll}7.66 & -0.20\end{array}\right]$} & {$\left[\begin{array}{ll}1.15 & -13.35\end{array}\right]^{T}$} \\
\hline$W_{9}$ & {$\left[\begin{array}{ll}4.70 & -0.15\end{array}\right]$} & {$\left[\begin{array}{ll}1.01 & -18.02\end{array}\right]^{T}$} \\
\hline$W_{8}$ & {$\left[\begin{array}{ll}8.64 & -0.21\end{array}\right]$} & {$\left[\begin{array}{ll}0.99 & -21.38\end{array}\right]^{T}$} \\
\hline$W_{7}$ & {$\left[\begin{array}{ll}5.19 & -0.15\end{array}\right]$} & {$\left[\begin{array}{ll}1.00 & -22.05\end{array}\right]^{T}$} \\
\hline$W_{6}$ & {$\left[\begin{array}{ll}13.66 & -0.20\end{array}\right]$} & {$\left[\begin{array}{ll}1.01 & -19.09\end{array}\right]^{T}$} \\
\hline$W_{5}$ & {$\left[\begin{array}{lll}13.04 & -0.19\end{array}\right]$} & {$\left[\begin{array}{ll}1.01 & -13.95\end{array}\right]^{T}$} \\
\hline
\end{tabular}

control input whenever a negative insulin delivery rate is required from the control law.

\section{Numerical Simulations}

Simulations have been carried out on a virtual patient with the following model parameters showing severe hyperglycemia and the state of frank Type 2 Diabetes Mellitus (refer to [21] for the details):

$$
\begin{array}{lll}
G_{b}=10.66 & I_{b}=49.29 & T_{i \text { max }}=0.236 \\
\gamma=3.205 & G^{*}=9 & \tau_{g}=24 \\
V_{G}=0.187 & K_{x i}=1.211 \cdot 10^{-2} & T_{g h}=0.003 \\
V_{I}=0.25 & K_{x g i}=3.11 \cdot 10^{-5} &
\end{array}
$$

The desired reference level is $G_{d e s}=5 \mathrm{mM}$. Let us consider a maximum bound $G_{\max }$ for plasma glycemia equal to $30 \mathrm{mM}$ : even if post-prandial glycemia could be rather high, especially for diabetic patients (and comparable to such an upper bound) simulations will show that such an upper bound is far larger than what we really obtain. The partition $W_{k}, k=0,1, \ldots, 30$ of the glucose interval (13) is set with $G_{k}=k \mathrm{mM}$ and

$$
a_{k+1}=b_{k}=0.5 m M, \quad k=0,1, \ldots, 29
$$

so that to have 30 intervals, all but the boundary ones $W_{0}$ and $W_{p}$ equally spaced with length $1 \mathrm{mM}$. Again, we stress the fact that very few intervals will be involved in the glucose evolution.

Let us consider first the task to regulate the patient glycemia at rest, that means without meal disturbances. In Table I the values of $K$ and $L$ are reported for regions $W_{k}$, $k=5, \ldots, 10$. Since no disturbance is present, parameter $\gamma$ does not play any role for this first set of simulations. According to the proposed control algorithm, the plasma glycemia reduces below the safety level of $6 \mathrm{mM}$ within the first 3/4 hours and becomes closer and closer to the desired level of glycemia within 6 hours, in a monotonically decreasing fashion: as a matter of fact only the 6 regions $W_{5}, \ldots, W_{10}$ are in fact involved, and no dangerous glucose oscillations appear. The observer is supposed to start with an initial error of $10 \%$ for both glycemia and insulinemia measurements. Simulated plasma glycemia is reported in Fig.1.

The second set of simulations is done by taking into account a meal administration, modeled by the presence of an unknown disturbance in the plasma glycemia dynamics. 


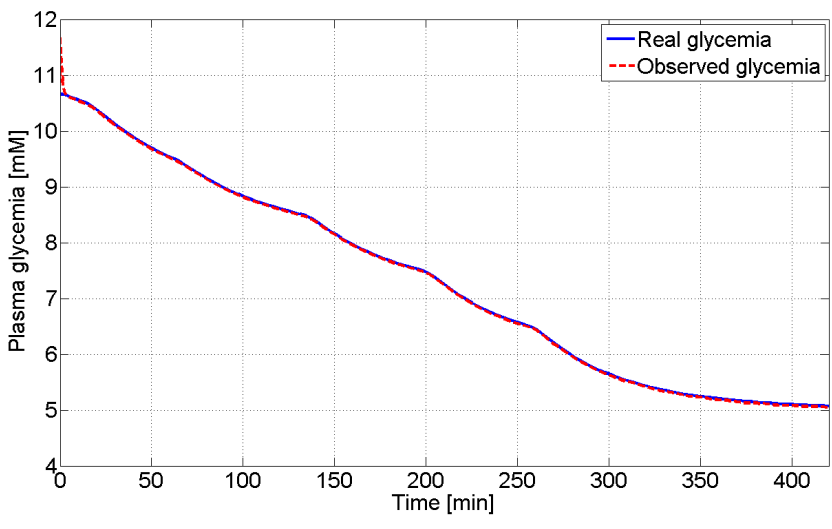

Fig. 1. Plasma glycemia for a closed loop system without disturbance.

TABLE II

CONTROL GAIN $K$ FOR $\gamma=\sqrt{800}$ AND $\gamma=\sqrt{10}$

\begin{tabular}{|c|c|c|}
\hline Regions & $K$ for $\gamma=\sqrt{800}$ & $K$ for $\gamma=\sqrt{10}$ \\
\hline$W_{10}$ & {$\left[\begin{array}{ll}423.9 & -1.1\end{array}\right]$} & {$\left[\begin{array}{ll}3274.7 & -3.8\end{array}\right]$} \\
\hline$W_{9}$ & {$\left[\begin{array}{lll}481.0 & -1.2\end{array}\right]$} & {$[2189.0-2.9]$} \\
\hline$\overline{W_{8}}$ & [333.3 -0.9$]$ & {$\left[\begin{array}{ll}1717.4 & -2.9\end{array}\right]$} \\
\hline$W_{7}$ & {$\left[\begin{array}{ll}1955.5 & -2.4\end{array}\right]$} & {$[3747.9-4.1]$} \\
\hline$W_{6}$ & {$\left[\begin{array}{ll}325.9 & -0.8\end{array}\right]$} & {$\left[\begin{array}{ll}5605.8 & -3.8]\end{array}\right.$} \\
\hline$W_{5}$ & {$\left[\begin{array}{lll}224.7 & -0.9\end{array}\right]$} & {$[2438.9-2.5]$} \\
\hline
\end{tabular}

The meal is administered at time $t=20 \mathrm{~min}$ : the rate of appearance of the glucose intake in the plasma compartment, the unknown disturbance $d(t)$, is taken from [4], according to proper unit conversions.

These kind of simulations are carried out by assuming that the patient's glycemia has already been controlled to track the desired value, so that the present simulations are made after the transient of the first class of simulations has vanished and the desired values $\left(G_{d e s}, I_{d e s}, u_{d e s}\right)$ in (12) have been asymptotically reached. In the spirit of the $H_{\infty}$ approach, two simulations are here reported, obtained by means of a couple of values of parameter $\gamma$. In Table II the values of the controller gain $K$ are reported for the different values of the linearization region and for different values of $\gamma$. The observer gains $L$ do not change from the values of Table I, exploited to synthesize the control law without the presence of the meal disturbance (indeed, the LMI (4) in Theorem 1 does not involve $L$ ).

Figs.2-3 report plasma glycemia and insulinemia, respectively, according to the exogenous insulin infusion rate pictured in Fig.4 and corresponding to a value of $\gamma$ equal to $\sqrt{800}$. The simulation considers the 7 hours after the meal administration. As it clearly appears, the maximum value of plasma glycemia is below $8.5 \mathrm{mM}$ and is reached within the first hour of simulation; two hours after the meal administration plasma glycemia is already below $7.5 \mathrm{mM}$ according to a monotonically decreasing behavior, that is considered an excellent result according to the efficacy criteria adopted to evaluate glucose controllers (see, e.g. [2] where excellent efficacy in the post-prandial state is assessed if after two hours of meal administration plasma glycemia is constrained below $8 \mathrm{mM}$ ). Notice that the spikes in the insulin infusion rate appear in correspondence of the time instant when plasma glycemia crosses one of the $W_{k}$ regions and the control parameters switch according to a new glucose target.

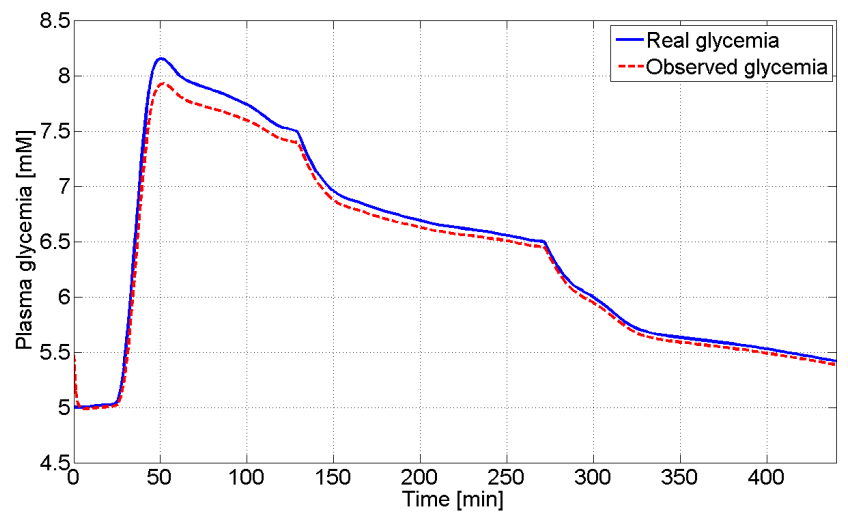

Fig. 2. Plasma glycemia for a closed loop system with $\gamma=\sqrt{800}$.

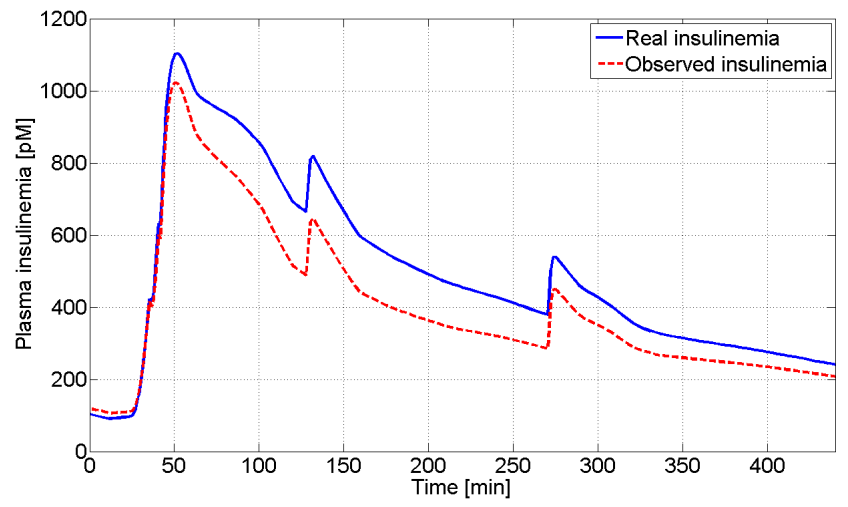

Fig. 3. Plasma insulinemia for a closed loop system with $\gamma=\sqrt{800}$.

Remark 5: By decreasing parameter $\gamma$ the disturbance is required to be more attenuated. Indeed, by setting $\gamma$ at $\sqrt{10}$ we obtain a further improvement of the disturbance rejection. Of course, in this latter case, a greater insulin administration is required at the beginning of the regulation.

\section{CONCLUSIONS}

A challenging task in model-based artificial pancreas is to cope with the many sources of uncertainties and disturbances that unavoidably affect the system, such as exercise, stress and meals.

In the spirit of the $H_{\infty}$ approach, a new control algorithm for the regulation of plasma glycemia is here presented, aiming to properly address the disturbances appearing in the glucose dynamics during a meal administration. Such an algorithm is based on LMIs for retarded systems, and makes use of a switching control law, with the switches depending on the current value of the glycemia. A partition of an interval of glycemia is used, and linearization of the RFDE describing the glucose-insulin system is considered 


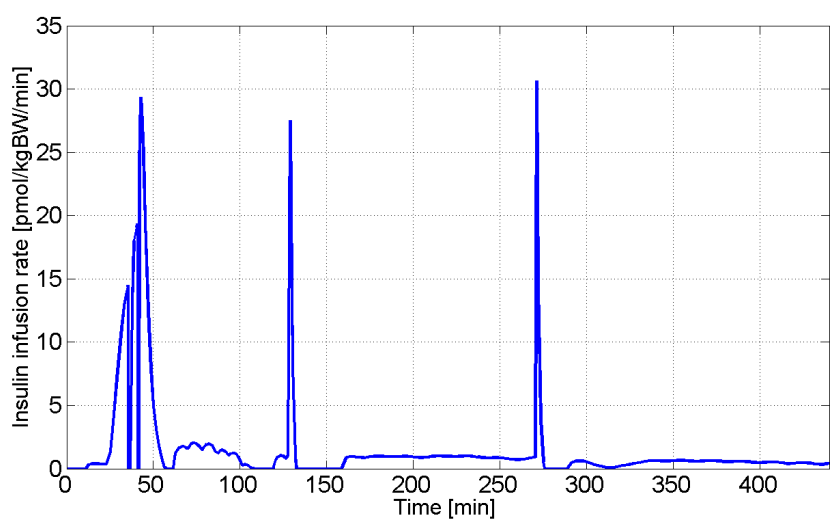

Fig. 4. Exogenous insulin administration for a closed loop system with $\gamma=\sqrt{800}$.

in each sub-interval. On the basis of the set of linear models, a set of controllers is found by means of standard LMIs. The simulations show very good performances of the controller, especially in cases modeling a meal admnistration. Future research efforts will concern the theoretical proof of convergence of the glycemia to the desired level, when the control law here presented is applied.

\section{REFERENCES}

[1] E.-K. Boukas, Z.-K. Liu, Deterministic and Stochastic Time Delay Systems, Birkhauser, Boston, 2002.

[2] L.J. Chassin, M.E. Wilinska and R. Hovorka, "Evaluation of glucose controllers in virtual environment: methodology and sample application," Artificial Intelligence in Medicine, 32, 171-181, 2004.

[3] Chee, F., Savkin, A.V., Fernando, T.L., and Nahavandi, S. (2005), "Optimal $H_{\infty}$ insulin injection control for blood glucose regulation in diabetic patients," IEEE Trans. on Biomed. Eng., 52, 1625-1631.

[4] C. Dalla Man, R.A. Rizza, C. Cobelli, "Meal simulation model of the glucose-insulin system," IEEE Trans. Biomed. Eng., 54, 1740-1749, 2007.

[5] Dua, P., Doyle, F.J., and Pistikopoulos, E.N. (2006), "Model-based blood glucose control for Type 1 diabetes via parametric programming," IEEE Trans. Biomedical Engineering, 53, 1478-1491.

[6] A. Germani, C. Manes, P. Pepe, "A Twofold Spline Approximation for Finite Horizon LQG Control of Hereditary Systems," SIAM Journal on Control and Optimization, 39(4), 1233-1295, 2000.

[7] K. Gu, V. L. Kharitonov and J. Chen, Stability of Time-Delay Systems, Control Engineering, Birkhaeuser, 2003.

[8] Hovorka, R., Canonico, V., Chassin, L.J., Haueter, U., MassiBenedetti, M., Federici, M.O., Pieber, T.R., Schaller, H.C., Schaupp, L., Vering, T., and Wilinska, M.E. (2004), "Nonlinear model predictive control of glucose concentration in subjects with type I diabetes," Physiological Measurements, 25, 905-920.

[9] M. Huang, J. Li, X. Song, H. Guo (2012), "Modeling impulsive injections of insulin: towards artificial pancreas," SIAM Journal of Applied Mathematics, 72(5), 1524-1548.

[10] H. Ito, P. Pepe, Z.-P. Jiang, "A small-gain condition for iISS of interconnected retarded systems based on Lyapunov-Krasovskii functionals", Automatica, Vol. 46, N. 10, pp. 1646-1656, 2010.

[11] I. Karafyllis, P. Pepe, and Z.-P. Jiang, "Global output stability for systems described by retarded functional differential equations: Lyapunov characterizations," European Journal of Control, Vol. 14, No. 6, pp. 516-536, 2008.

[12] I. Karafyllis, P. Pepe, and Z.-P. Jiang, "Input-to-output stability for systems described by retarded functional differential equations," $E$ ropean Journal of Control, Vol. 14, No. 6, pp. 539-555, 2008.

[13] L. Kovács, B. Kulcsár, A. György and Z. Benyó, "Robust servo control of a novel type 1 diabetic model," Optimal Control Applications and Methods, 32, 215-238, 2011.
[14] L. Kovács, B. Benyó, J. Bokor and Z. Benyó, "Induced $L_{2}$-norm minimization of glucose-insulin system for Type I diabetic patients," Computer Methods and Programs in Biomed., 102, 105-118, 2011.

[15] L. Kovács, P. Szalay, Z. Almassy, L. Barkai, "Applicability Results of a Nonlinear Model-Based Robust Blood Glucose Control Algorithm," Journal of Diabetes Science and Technology, 7(3), 708-716, 2013.

[16] L. Magni, D.M. Raimondo, C. Dalla Man, G. De Nicolao, B. Kovatchev and C. Cobelli, "Model predictive control of glucose concentration in type I diabetic patients: An in silico trial," Biomedical Signal Processing and Control, 4, 338-346, 2009.

[17] Makroglou, A., Li, J., and Kuang, Y. (2006), "Mathematical models and software tools for the glucose-insulin regulatory system and diabetes: an overview," Applied Numerical Mathematics, 56, 559-573.

[18] S. Niculescu and K. Gu, Advances in Time-Delay Systems, Lecture Notes in Computational Science and Engineering, Springer, 2004.

[19] P. Palumbo, S. Panunzi, A. De Gaetano, "Qualitative behavior of a family of delay differential models of the glucose insulin system," Discrete and Continuous Dynamical Systems - Series B, 7, 399-424, 2007.

[20] P. Palumbo, P. Pepe, S. Panunzi, A. De Gaetano, "Robust Closed-Loop Control of Plasma Glycemia: a Discrete-Delay Mode," Discrete and Continuous Dynamical Systems - Series B, 12(2), 455-468, 2009.

[21] P. Palumbo, P. Pepe, S. Panunzi, A. De Gaetano, "Time-delay modelbased control of the glucose-insulin system, by means of a state observer," Eur. J. Control, 18(6), 591-606, 2012.

[22] P. Palumbo, G. Pizzichelli, S. Panunzi, P.Pepe, A. De Gaetano, "Observer-based closed-loop control for the glucose-insulin system: local Input-to-State Stability with respect to unknown meal disturbances," Proc. American Control Conference, Washington, DC, USA, 1754-1759, 2013.

[23] P. Palumbo, G. Pizzichelli, S. Panunzi, P.Pepe, A. De Gaetano, "Tests on a virtual patient for an observer-based, closed-loop control of plasma glycemia," Proc. 50th IEEE Conf. Decision and Control, Orlando, USA, 6936-6941, 2011.

[24] S. Panunzi, P. Palumbo, A. De Gaetano, "A discrete single delay model for the Intra-Venous Glucose Tolerance Test," Theoretical Biology and Medical Modelling, 4(35), 1-16, 2007.

[25] Parker, R.S., Doyle III, F.J., Ward, J.H., and Peppas, N.A. (2000), "Robust $H_{\infty}$ glucose control in diabetes using a physiological model," AIChE Journal, 46, 2537-2549.

[26] P. Pepe, and Z.-P. Jiang, "A Lyapunov-Krasovskii methodology for ISS and iISS of time-delay systems," Systems \& Control Letters, Vol. 55, No. 12, pp. 1006-1014, 2006.

[27] P. Pepe, "The problem of the absolute continuity for LyapunovKrasovskii functionals," IEEE Transactions on Automatic Control, Vol. 52, No. 5, pp. 953-957, 2007.

[28] C. Scherer, S. Weiland, Linear Matrix Inequalities in Control, Lecture Notes, Delft Center for Systems and Control, Delft, The Nederlands, 2004.

[29] Ruiz-Velázquez, E., Femat, R., and CamposDelgado, D.U. (2004), "Blood glucose control for type I diabetes mellitus: a robust $H^{\infty}$ tracking problem," Control Engineering Practice, 12, 1179-1195. 